\title{
Comment l'objectif de maîtrise des flux de polluants est-il traduit dans les critères de gestion à l'amont des eaux pluviales ? - Analyse des pratiques en France et à l'international
}

\author{
Sage Jérémie ${ }^{1}$, Gromaire Marie-Christine ${ }^{1}$, Berthier Emmanuel ${ }^{2}$
}

${ }^{1}$ Laboratoire Eau Environnement Systèmes Urbains - ENPC, 6-8 av. Blaise pascal, 774555

Champs sur Marne, France, jeremie.sage @leesu.enpc.fr, gromaire@ @leesu.enpc.fr

${ }^{2}$ DRIEA/CETE Ile-de-France, Département Ville Durable / Unité Hydrologie - Gestion des eaux pluviales, 12 rue Teisserenc de Bort, 78197 Trappes Cedex, emmanuel.berthier@developpement-durable.gouv.fr

\section{Thème : Réglementation, Gestion à la source de la pollution des eaux pluviales Mots clés: Gestion, Eaux pluviales urbaines, Critères, Réglementation, Flux de polluants Orateur : Jérémie Sage}

Les flux de polluants associés aux eaux pluviales urbaines sont un facteur majeur de dégradation des milieux aquatiques superficiels dont la gestion est devenue un réel enjeu écologique au cours des dernières décennies. De nombreuses solutions techniques ont par ailleurs été développées afin de limiter la formation du ruissellement à l'amont des bassins versants urbains. Néanmoins, alors que la plupart des pays ou collectivités aspirent à la maîtrise de cette pollution, les réglementations ou prescriptions données aux aménageurs demeurent hétérogènes et intègrent rarement des critères directement liés à la maîtrise des flux de polluants. Ainsi, alors qu'en France règlements d'assainissement ou SDAGE fixent généralement un débit de fuite, d'autres critères de gestion sont employés à l'étranger.

Ce travail vise donc dans un premier temps à identifier puis analyser les différents critères de gestion donnés aux aménageurs. En se basant sur plus d'une cinquantaine de références réglementaires ou de guides de gestion des eaux pluviales à l'international, quatre grandes catégories de critères ont pu être identifiées :

- La régulation des débits rejetés vers les réseaux ou les milieux récepteurs

- L'interception d'un volume « à traiter » (avec restitution éventuelle)

- L'abattement d'une certaine hauteur d'eau (infiltrée ou évapotranspirée)

- La réduction des flux de polluants (respect d'un seuil de concentration ou abattement des masses annuelles pour un polluant données)

Dans les différents cas étudiés, l'analyse du contexte institutionnel révèle que les contraintes données aux aménageurs relèvent à la fois de principes généraux (enjeux régionaux de préservation des milieux) et d'exigences subsidiaires inhérentes aux spécificités locales (capacité des réseaux ou enjeu écologique particulier). L'objectif initial de réduction des flux de polluants est donc très souvent adapté à des échelles de responsabilité plus décentralisées ce qui explique en grande partie la diversité des critères donnés aux aménageurs.

A cette hétérogénéité dans les critères est également associée une hétérogénéité dans les outils permettant leur mise en œuvre (choix et conception des techniques alternatives) : l'étude 
s'intéresse donc également à la manière dont les différents critères sont intégrés aux références techniques et facilitent ou non l'adoption de solutions efficaces de gestion du ruissellement. Ces derniers conditionnent en effet fortement le choix des solutions techniques. Il convient donc de distinguer la pertinence théorique des différents critères (« correspondent-ils a priori à l'objectif de maîtrise des flux de polluants ?»), de leur capacité à être traduits en termes de conception ou de dimensionnement.

Cette étude suggère alors que la mise en œuvre de pratiques de gestion efficaces et à moindre impact des eaux de ruissellement ne dépend pas tant de la nature des critères donnés aux aménageurs que des outils permettant leur prise en compte pour le choix et la conception des techniques alternatives. 